\title{
Composite Position
}

National Cancer Institute

\section{Source}

National Cancer Institute. Composite Position. NCI Thesaurus. Code C43437.

The location in the compositeSequence target's sequence to which a source

compositeSequence maps. The association to MismatchInformation allows the specification, usually for control purposes, of deviations from the CompositeSequence's BioMaterial. (caMAGE) 